\title{
Is Regression after Neoadjuvant Chemotherapy for Locally Advanced Breast Cancer Different in Sentinel and Non-sentinel Nodes?
}

\author{
Gábor Cserni $^{1,2}$ (1) $\cdot$ Tamás Zombori $^{2} \cdot$ Xavier Andreu $^{3} \cdot$ Simonetta Bianchi $^{4}$. \\ Peter Regitnig $^{5}$ - Isabel Amendoeira ${ }^{6}$ • Davide Balmativola ${ }^{7}$ - Anikó Kovács ${ }^{8}$. \\ Alicia Cordoba ${ }^{9}$ - Angelika Reiner ${ }^{10}$ • Janina Kulka ${ }^{11}$ - Handan Kaya ${ }^{12}$. \\ Inta Liepniece-Karele ${ }^{13}$. Cecily Quinn ${ }^{14}$ - Bence Kővári ${ }^{2}$
}

Received: 3 March 2017 / Accepted: 3 April 2017 /Published online: 8 April 2017

(C) Arányi Lajos Foundation 2017

\begin{abstract}
Tumor draining sentinel lymph nodes (SLNs) are the sites of selective changes as compared to non-SLNs. They show features of tumor-reactive lymphadenopathy, including increased total number of functional blood vessels, but a relative immunosuppressed status has also been described in them. We explored the hypothesis of a selective regression or non-regression in SLNs versus non-SLNs in 142 patients with 110 estrogen receptor-positive and 32 estrogen receptornegative tumors undergoing both SLN biopsy and axillary lymph node dissection after neoadjuvant therapy by assessing the tumoral (metastatic) and regression statuses of SLNs and non-SLNs separately. Of the 89 cases with signs of nodal regression, 22 cases $(25 \%)$ were in favor of a selective nonregression in SLNs, 18 cases (20\%) were supportive of a selective and more pronounced regression in the SLNs and the remaining showed equal degrees of regression or non-
\end{abstract}

Gábor Cserni

cserni@freemail.hu

1 Department of Pathology, Bács-Kiskun County Teaching Hospital, Nyiri ut 38, Kecskemét 6000, Hungary

2 Department of Pathology, University of Szeged, Állomás u 1, Szeged 6725, Hungary

3 Department of Pathology, Corporació Sanitària Parc Taulí, University Autònoma Barcelona, 08202 Sabadell, Spain

4 Division of Pathological Anatomy, Department of Surgery and Translational Medicine, AOU Careggi, Largo G. A. Brambilla 3, 50134 Florence, Italy

5 Institute of Pathology, Medical University of Graz, Auenbruggerplatz 25, A-8036 Graz, Austria

6 Laboratório de Anatomia Patológica, Centro Hospitalar de São João e IPATIMUP, 4440-563 Porto, Portugal regression in SLNs and non-SLNs. The results indicate that there is no obvious difference in the degree of regressive histological changes shown by SLNs and NSLNs. Therefore, this phenomenon may not be a major contributor to the higher false negative rate of SLN biopsy after neoadjuvant treatment.

Keywords Sentinel lymph node $\cdot$ Non-sentinel lymph node Breast cancer $\cdot$ Neoadjuvant therapy $\cdot$ Selective regression

\section{Introduction}

Lymph nodes are not identical. The sentinel lymph nodes (SLN) are the first lymph nodes draining the lymph from an anatomical site and therefore, in case of a cancer in the given area, the SLNs are the first involved during the lymphatic

7 Candiolo Cancer Institute - Fondazione del Piemonte per l'Oncologia (FPO), IRCCS, Str. Prov. 142, km 3.95, 10060 Candiolo, To, Italy

8 Department of Clinical Pathology and Genetics, Sahlgrenska University Hospital, Gula stråket 8, 41345 Gothenburg, Sweden

9 Department of Pathology, Complejo Hospitalario de Navarra, Irunlarrea 3, 31008 Pamplona, Navarra, Spain

10 Pathologisch-Bakteriologisches Institut, Donauspital am SMZO, Langobardenstraße, 122 Vienna, Austria

11 2nd Department of Pathology, Semmelweis University Budapest, Üllöi út 93, Budapest H-1091, Hungary

12 Department of Pathology, Marmara University, School of Medicine, Istanbul, Turkey

13 Pathology Centre, Riga East Clinical University Hospital, Hipokrata St 2, Riga LV-1038, Latvia

14 School of Medicine, University College Dublin, St. Vincent's University Hospital, Elm Park, Dublin 4, Ireland 
spread of cancer. The non-SLNs of the same anatomic region develop metastases only subsequently. Development of metastasis is not the only change known to occur in tumor draining lymph nodes. Tumor-reactive lymphadenopathy (TRL) is a complex reaction of lymph nodes, which develops before the arrival of the metastatic tumor cells and comprises morphological and functional changes. Enlargement of the tumor draining lymph nodes (also induced by needle biopsy of the primary cancer) has helped axillary four-node sampling [1], and may explain the possible overlap of axillary node samples and sentinel nodes [2]. As concerns the morphological alterations, previous publications found increased total number of functional blood vessels and lymphatic vessels/sinuses in tumor draining lymph nodes. Furthermore, some authors have described dilation of functional blood vessels with structural remodeling and endothelial cell proliferation of high endothelial venules [3]. Functional changes, such as increased blood perfusion and immunological differences, like the alterations in $\mathrm{CD} 28$ and $\mathrm{CD} 3 \zeta$ expression of CD4+ and CD8+ T-lymphocytes $[4,5]$ or the reduction in density and dendritic complexity of antigen presenting paracortical dendritic cells [6] were also observed in tumor draining lymph nodes. Tumor-induced immune modulation of the SLNs versus the non-SLNs seems to act in favor of a reduced anti-tumoral immune reaction [6].

The above-mentioned factors may hypothetically alter the neoadjuvant therapy induced reactions in tumor-draining SLNs compared to lymph nodes without direct connection with the primary carcinoma, i.e. non-SLNs. Enhanced blood flow and enriched vasculature may increase the load of chemotherapeutics or targeted cancer therapy agents (e.g. hormone receptor modulators or trastuzumab) in lymph nodes developing TRL. A preferential regression in SLNs might influence the false negative rate of SLN biopsy after primary systemic treatment, by resulting in complete regression in SLNs with remaining tumor burden in non-SLNs. Alternatively an immunosuppressed status of the SLNs as compared to non-SLNs could lead to less effect from cytotoxic therapeutics. A selective regression or non-regression may also alter the staging effect of the removal of radioactive seed localized nodes that proved to be positive before the initiation of primary systemic treatment [7].

Along this hypothesis, differences in the degree of regression induced by neoadjuvant therapy in the SLNs (tumor draining lymph nodes) and in non-SLNs (non-tumor draining lymph nodes) were looked for in a series of patients who underwent SLN biopsy and axillary lymph node dissection (ALND) following neoadjuvant systemic therapy for breast cancer.

\section{Materials and Methods}

In this retrospective study, lymph node tissue sections of 142 female breast cancer patients staged with SLN biopsy and ALND after receiving neoadjuvant therapy were used from the archives of the authors' institutions. Inclusion criteria for enrollment were any type of histologically verified invasive primary breast cancer treated with neoadjuvant systemic therapy, the successful identification of at least one SLN and ALND following SLN biopsy. SLNs were identified according to the routine procedure of the given institutions, and involved intra- or peritumoral or subareolar injection of a $99 \mathrm{mTc}$ labelled colloidal albumin and/or Patent blue dye in vivo. ALND was performed either routinely as part of a validation of SLN biopsy after neoadjuvant treatment or because of metastatic disease identified in the SLNs.

Tissues were fixed in buffered formalin and embedded in paraffin. Three to four-micrometer-thick whole tissue sections were used for the standard hematoxylineosin staining. The presence and degree of regression were evaluated in all metastatic and non-metastatic lymph nodes, SLNs and non-SLNs separately, using the nodal regression (NR) grading recommended by the European guidelines [8]. These results were used to categorize the cases, based on whether they supported the theory that regression in SLNs may be greater than in NSLNs or not (Table 1). The table reflects the nodes with the best/highest degree of regression for each case.

No patient identity related data were required or used, disease outcome was indifferent for the study purposes, and this retrospective non-interventional setting had no influence on treatment. Such a protocol does not imply an ethical approval in most of the participating institutions, but the Ethical Committee and Data Safety Manager of Bács-Kiskun County Teaching Hospital, where the study was initiated, were consulted and no ethical concerns were raised (Reference Number: 4/170125).

\section{Results}

The mean ( \pm S.D.) age of the patients was $50.2( \pm 11.0)$ years. A median of 2 SLNs (range: 1-8) and 14 nonSLNs (range: 2-42) were removed from the patients. The neoadjuvant treatment involved hormonal therapy with aromatase inhibitors in 10 cases, whereas the remaining patients received chemotherapies. There were 110 estrogen receptor (ER) positive tumors (including 25 human epidermal growth factor receptor 2 - HER2 positive cases), and of the 32 ER negative tumors, 12 were HER2 positive by immunohistochemistry or in situ hybridization. Complete primary tumor regression with or without residual in situ carcinoma was seen in 24 cases, and no signs of regression were reported in 16 primary cancers. No nodal regression at all was 
Table 1 Categorization of the results on the basis of possible variations in the distribution of metastases and regression and their relation to the theory of a possible selective regression pattern in SLNs

\begin{tabular}{|c|c|c|c|c|c|c|}
\hline $\mathrm{SN}$ metastasis & $\mathrm{SN}$ regression & NSN metastasis & NSN regression & Category & Relation to theory & No. of cases \\
\hline \multirow[t]{10}{*}{ Yes } & \multirow[t]{6}{*}{ Yes } & \multirow[t]{2}{*}{ No } & Yes & A & Against & 9 \\
\hline & & & No & $\mathrm{B}$ & Indifferent & 15 \\
\hline & & \multirow[t]{4}{*}{ Yes } & Yes $(=\mathrm{SN})$ & $\mathrm{CO}$ & Indifferent & 8 \\
\hline & & & Yes $(<\mathrm{SN})$ & $\mathrm{C} 1$ & In favor & 11 \\
\hline & & & Yes $(>\mathrm{SN})$ & $\mathrm{C} 2$ & Against & 5 \\
\hline & & & No & $\mathrm{D}$ & In favor & 6 \\
\hline & \multirow[t]{4}{*}{ No } & \multirow[t]{2}{*}{ No } & Yes & $\mathrm{E}$ & Against & 3 \\
\hline & & & No & $\mathrm{F}$ & Indifferent & 24 \\
\hline & & \multirow[t]{2}{*}{ Yes } & Yes & G & Against & 5 \\
\hline & & & No & $\mathrm{H}$ & Indifferent & 18 \\
\hline \multirow[t]{8}{*}{ No } & \multirow[t]{4}{*}{ Yes } & \multirow[t]{2}{*}{ No } & Yes & I & Indifferent & 11 \\
\hline & & & No & $\mathrm{J}$ & Indifferent & 2 \\
\hline & & \multirow[t]{2}{*}{ Yes } & Yes & $\mathrm{K}$ & In favor & 1 \\
\hline & & & No & $\mathrm{L}$ & In favor & 0 \\
\hline & \multirow[t]{4}{*}{ No } & \multirow[t]{2}{*}{ No } & Yes & M & False negative SLN & 7 \\
\hline & & & No & $\mathrm{N}$ & Indifferent & 9 \\
\hline & & \multirow[t]{2}{*}{ Yes } & Yes & $\mathrm{O}$ & False negative SLN & 6 \\
\hline & & & No & $\mathrm{P}$ & False negative SLN & 2 \\
\hline
\end{tabular}

evidenced in 19 node-positive cases, whereas 55 cases had at least one metastatic lymph node without evidence of regression (NR4). The metastasis and regression status of the analyzed cases are summarized in Table 1.

Categories B, C0, F, H, I, J and N represented 87/142 cases including 40 node-positive cases without evidence of regression in the lymph nodes (Table 1), and were indifferent towards the tested hypothesis. Of the 89 cases with signs of nodal regression, the 22 cases in categories $\mathrm{A}, \mathrm{C} 2, \mathrm{E}$ and $\mathrm{G}$, where the regression was of greater degree in non-SLNs (22/ 89 cases, $25 \%$ ) were in favor of a selective non-regression in SLNs. Only 18/89 cases (20\%) fell into categories C1, D or K, and were in support of a selective and more pronounced regression in the SLNs.

\section{Discussion}

The practice of SLN biopsy in locally advanced breast cancer patients receiving neoadjuvant systemic treatment is still somewhat controversial. Often the SLN biopsy was performed before primary systemic therapy or was not performed because of previously evidenced metastatic lymph nodes. The required cases were relatively rare in our archives, and the present study should be regarded as an exploration of the theory of a potential selective regression (or non-regression) in SLNs.
There are some reservations regarding the possible conclusion of the study. The methods of SLN biopsy were obviously not uniform, but were the ones applied and relied on in clinical practice at the given institution. The nature of the therapeutic agents used for primary systemic treatment could not be taken into account, as there was a wide variation in the protocols and drugs used, and the low case numbers would not have made any treatment-specific analysis possible. Altogether the case numbers are relatively small, however, the distribution of the cases did not point in favor or against a selective regression (or non-regression) in SLNs. Finally, fibrotic foci in the lymph nodes were interpreted as signs of regression, but these signs are not specific, and other processes may lead to the same morphological features. However, in the setting of neoadjuvant therapy, this latter is the most likely cause for these morphological changes, and therefore these were uniformly interpreted as signs of regression.

The evidence gathered from the limited number of cases available suggests that despite the attractiveness of a preferential regression (or non-regression) in tumor draining lymph nodes (SLNs which seem to be immunosuppressed and at the same time are the sites of TRL), there is no obvious difference in the degree of regressive histological changes shown by SLNs and NSLNs. Therefore, this phenomenon may not be a major contributor to the somewhat higher false negative rate of SLN biopsy after neoadjuvant treatment [9]. 
Acknowledgements The study was supported by the National Research, Development and Innovation Office grant GINOP-2.3.2-152016-00020. The content of the communications has not been influenced by this support.

\section{References}

1. Greenall MJ (1995) Why I favour axillary node sampling in the management of breast cancer. Eur J Surg Oncol 21:2-5

2. Cserni G (1999) Estimating the overlap between sentinel lymph nodes and axillary node samples in breast cancer. Pathol Oncol Res 5:129-133

3. Qian CN, Berghuis B, Tsarfaty G, Bruch M, Kort EJ, Ditlev J, Tsarfaty I, Hudson E, Jackson DG, Petillo D, Chen J, Resau JH, Teh BT (2006) Preparing the "soil": the primary tumor induces vasculature reorganization in the sentinel lymph node before the arrival of metastatic cancer cells. Cancer Res 66:10365-10376

4. Schüle JM, Bergkvist L, Håkansson L, Gustafsson B, Håkansson A (2004) CD28 expression in sentinel node biopsies from breast cancer patients in comparison with CD3-zeta chain expression. J Transl Med 2:45. doi:10.1186/1479-5876-2-45

5. Schüle J, Bergkvist L, Håkansson L, Gustafsson B, Håkansson A (2002) Down-regulation of the CD3-zeta chain in sentinel node biopsies from breast cancer patients. Breast Cancer Res Treat 74:33-40

6. Cochran AJ, Huang RR, Lee J, Ikatura E, Leong SP, Essner R (2006) Tumour-induced immune modulation of sentinel lymph nodes. Nat Rev Immunol 6:659-670

7. Straver ME, Loo CE, Alderliesten T, Rutgers EJ, Vrancken Peeters MT (2010) Marking the axilla with radioactive iodine seeds (MARI procedure) may reduce the need for axillary dissection after neoadjuvant chemotherapy for breast cancer. Br J Surg 97:1226-1231

8. Wells CA, Amendoeira I, Bellocq JP et al (2012) S2: pathology update. Quality assurance guidelines for pathology. In: Perry N, Broeders $\mathrm{M}$, de Wolf $\mathrm{C}$ et al (eds) European guidelines for quality assurance in breast cancer screening and diagnosis, 4th edn, supplements. European Commission, Office for Official Publications of the European Union, Luxembourg, pp 73-120

9. Mocellin S, Goldin E, Marchet A, Nitti D (2016) Sentinel node biopsy performance after neoadjuvant chemotherapy in locally advanced breast cancer: a systematic review and meta-analysis. Int $\mathrm{J}$ Cancer 138:472-480 